Bulletin of the Seismological Society of America. Vol. 49, No. 4, pp. 355-364 October, 1959

\title{
RAYLEIGH-WAVE EVIDENCE FOR THE LOW-VELOCITY ZONE IN THE MANTLE
}

\author{
By H. Takeuchi, F. Press, and N. Kobayashi
}

\begin{abstract}
Variational calculus methods are applied to the problem of dispersion of mantle Rayleigh waves. In the present paper we have worked two models. One is Gutenberg's model with a low-velocity layer around $150 \mathrm{~km}$. depth. The other is a Jeffreys-Bullen model modified above $200 \mathrm{~km}$. depth so as to join smoothly to the explosion-determined velocities just under the Mohorovičie discontinuity. No low-velocity layer is assumed in this model. Both models give almost identical theoretical dispersion curves which agree well with the Ewing-Press observations of mantle Rayleigh waves for periods longer than $250 \mathrm{sec}$. This result means that the minimum group velocity at about 250 sec. is mainly due to a sharp increase of shear velocity at about $400 \mathrm{~km}$. depth, which is a common feature for the two models. For periods shorter than 250 sec. Gutenberg's model gives results concordant with the observations. The modified Jeffreys-Bullen model disagrees significantly with the observations. This demonstrates the existence of a low-velocity layer in the upper mantle.
\end{abstract}

\section{INTRODUCTION}

DATA from diverse sources confirm Gutenberg's conclusion (1953) that a low-velocity zone occurs in the mantle at depths of 100-200 km. Amplitude variations with distance of seismic waves from nuclear explosions are in substantial agreement with the data for earthquake-generated waves which are the basis of Gutenberg's results. (See verbatim proceedings of the Geneva Conference of Experts to study methods of detecting violations of a possible agreement on the suspension of nuclear tests, available at United Nations, New York.) Press and Ewing (1956) and Landisman and Satô (1958) believe that $G$-wave velocity data require the existence of a low-velocity zone. Press (1959) demonstrates that the zone is present under continents and oceans and suggests that it may be the source of the primary basaltic magma and that it accounts for the long-period nature of $\mathrm{S}$ waves. It is our purpose in this paper to show that mantle Rayleigh-wave dispersion provides additional evidence for the worldwide existence of this zone. We further show how variational techniques offer a relatively rapid method for deriving theoretical dispersion curves for any variation of density and elasticity with depth. This procedure may be suffciently precise for some problems.

\section{General Theory}

The problem of wave propagation in media with variable velocity has been studied by many authors. (See Ewing et al., 1957, chap. vii.) From the mathematical point of view, the problem is stated as follows: Taking $x$ and $z$ axes in horizontal and vertically downward directions, respectively, and assuming that the corresponding displacements $(u, w)$ are proportional to $e^{\imath k(x-C t)}$, we have the following equations of motion:

Manuscript received for publication February 17, 1959. 


$$
\begin{aligned}
\mu \frac{d^{2} u}{d z^{2}}+\frac{d \mu}{d z} \frac{d u}{d z}+k^{2}\left\{\rho c^{2}-(\lambda\right. & +2 \mu)\} u \\
& +i k\left\{(\lambda+\mu) \frac{d w}{d z}+\frac{d \mu}{d z} w\right\}=0 \\
(\lambda+2 \mu) \frac{d^{2} w}{d z^{2}}+\frac{d}{d z}(\lambda+2 \mu) \frac{d w}{d z}+ & k^{2}\left(\rho c^{2}-\mu\right) w \\
& +i k\left\{(\lambda+\mu) \frac{d u}{d z}+\frac{d \lambda}{d z} u\right\}=0
\end{aligned}
$$

where density $\rho$ and elastic constants $\lambda$ and $\mu$ are assumed to be some functions of $z$. At the free surface $z=0$ we must have vanishing stress

$$
i k w+\frac{d u}{d z}=0=(\lambda+2 \mu) \frac{d w}{d z}+i k \lambda u
$$

and at discontinuity interfaces within the medium, if any, displacements and stresses

$$
u, w, \mu\left(i k w+\frac{d u}{d z}\right) \text { and }(\lambda+2 \mu) \frac{d w}{d z}+i k \lambda u
$$

must be continuous. This is an eigen-value problem in which we must determine the eigen-value $C$ (phase velocity) as a function of $k$ (wave number) to obtain the dispersion equation corresponding to the medium. For the precision required it is prohibitively difficult to solve this eigen-value problem by trial-and-error methods. Jeffreys (1935) first suggested the application of Rayleigh's principle to problems of this kind. We shall use an extension of this method which yields eigen-values with greater precision. A variational equation equivalent to (1)-(3) was obtained by Jeffreys. Putting $U=i u$ and making some rearrangements reduces his equation to

$$
\begin{gathered}
C^{2} \int_{0}^{+\infty} \rho(U \delta U+w \delta w) d(k z) \\
-\int_{0}^{+\infty} \lambda\left\{U \delta U+\frac{d w}{d(k z)} \frac{d \delta w}{d(k z)}+\frac{d w}{d(k z)} \delta U+U \frac{d \delta w}{d(k z)}\right\} d(k z) \\
-\int_{0}^{+\infty} \mu\left\{2 U \delta U+w \delta w+\frac{d U}{d(k z)} \frac{d \delta U}{d(k z)}+2 \frac{d w}{d(k z)} \frac{d \delta w}{d(k z)}\right. \\
\left.-\frac{d U}{d(k z)} \delta w-w \frac{d \delta U}{d(k z)}\right\} d(k z)=0
\end{gathered}
$$

We choose $k z$ rather than $z$ as an independent variable, for reasons which will be given shortly. In the following, we assume no discontinuity within the medium and thus disregard the boundary condition (3). If there is a discontinuity, we replace it 
by a thin transitional zone in which the density and elastic constants change rapidly and continuously. As the trial vector function $\vec{u}=(U, w)$, we take a linear combination of $\vec{u}(m, k z)$, each satisfying the remaining boundary condition (2). Thus, we have

$$
\vec{u}=\sum_{m} E_{m} \vec{u}(m, k z)
$$

where $E_{m}$ is some undetermined constant.

The exact solution of the Rayleigh-wave problem for a uniform medium will suggest the following trial functions:

$$
\begin{aligned}
& U=e^{-\alpha k z}-A e^{-\beta k z} \\
& w=B e^{-\alpha k z}-D e^{-\beta k z}
\end{aligned}
$$

The boundary conditions (2) yield the following two relations among $\alpha, \beta, A, B$, and $D$.

$$
\begin{aligned}
& B-D+\alpha-A \beta=0 \\
& B \alpha-D \beta+\frac{\lambda_{0}}{\lambda_{0}+2 \mu_{0}}(A-1)=0
\end{aligned}
$$

where $\lambda_{0}$ and $\mu_{0}$ are $\lambda$ and $\mu$ at the free surface $z=0$. In the ordinary Rayleigh-wave problem of a uniform medium for which $\lambda_{0}=\mu_{0}$, we have

$$
\begin{aligned}
& \alpha=\alpha_{0}=0.84748658, \quad \beta=\beta_{0}=0.39331990, \quad A=A_{0}=0.57735027 \\
& B=B_{0}=0.84748658, \quad D=D_{0}=1.46788981
\end{aligned}
$$

Assuming $\lambda_{0}=\mu_{0}$, we shall take

$$
\alpha=m \alpha_{0}, \quad \beta=m \beta_{0}, \quad A=A_{0}
$$

for $\vec{u}(m, k z)$ in (5), where $m$ is some numerical constant. The corresponding $B$ and $D$ are, by (7),

$$
\begin{aligned}
& B=0.53728499 m+0.31020163(1 / m) \\
& D=1.15768822 m+0.31020163(1 / m)
\end{aligned}
$$

Understanding that the variation $\delta$ in (4) is applied only to the undetermined constants $E_{m}$, we get from (4) $m$ simultaneous linear equations for $E_{m}$. The coefficient of $E_{m}$ in the $m^{\prime}$ th equation will be equal to the left-hand side of (4) with $\vec{u}$ and $\delta \vec{u}$ replaced by $\vec{u}(m, k z)$ and $\vec{u}\left(m^{\prime}, k z\right)$, respectively. Putting the determinant formed by the coefficients thus obtained equal to zero gives an algebraic equation to determine $C$. The reason why we chose $k z$ rather than $z$ as an independent variable is now clear. In this way, almost all of the computations can be made independently of the wave number $k$, which need be considered only when we come to the distributions of $\rho, \lambda$, and $\mu$ in the $k z$ space. 
In our numerical computation, we use $\vec{u}(1, k z), \vec{u}(2, k z)$, and $\vec{u}(4, k z)$. Numerical results obtained by using $\vec{u}(1, k z) ; \vec{u}(1, k z)$, and $\vec{u}(2, \mathrm{kz}) ; \vec{u}(1, k z), \vec{u}(2, k z)$, and $\vec{u}(4, k z)$, will be called the first, second, and third approximations, respectively. In order to test our method, we first work out the example given by Jeffreys for a homogeneous superficial layer over a homogeneous half-space. The density and elasticities in the lower medium are respectively $5 / 4$ and $20 / 9$ times those in the

TABLE 1

\begin{tabular}{|c|c|c|c|c|c|c|}
\hline & \multirow{2}{*}{ Depth } & \multirow{2}{*}{ Density } & \multicolumn{2}{|c|}{ Jeffreys } & \multicolumn{2}{|c|}{ Gutenberg } \\
\hline & & & $V_{\mathbf{P}}$ & $V_{S}$ & $V_{\mathbf{p}}$ & $V_{\mathcal{S}}$ \\
\hline & $\mathrm{km}$. & $\mathrm{gm} / \mathrm{cm}$. & $\mathrm{km} / \mathrm{sec}$. & $\mathrm{km} / \mathrm{sec}$ & $\mathrm{km} / \mathrm{sec}$ & $\mathrm{km} / \mathrm{sec}$. \\
\hline 33 & & 332 & 775 & 435 & $\ldots$ & . \\
\hline 60 & . $\quad$. & & & & 8.15 & 46 \\
\hline 100 & & 338 & 795 & 445 & 80 & 44 \\
\hline 150 & & & & & 7.85 & 435 \\
\hline 200 & . & 347 & 8.26 & 460 & 805 & 44 \\
\hline 300 & . . . & 3.55 & 858 & 476 & 85 & 46 \\
\hline 400 & & 363 & 893 & 4.94 & 90 & 495 \\
\hline 500 & . & 389 & 966 & 5.32 & 96 & 53 \\
\hline 600 & & 4.13 & 1024 & 566 & 101 & 56 \\
\hline 700 & & 4.33 & 1067 & 593 & 105 & 5.9 \\
\hline 800 & & 4.49 & 1101 & 613 & 109 & 6.15 \\
\hline 900 & & 4.60 & 11.25 & 627 & 113 & 6.3 \\
\hline 1,000 & & 468 & 1143 & 636 & 114 & 635 \\
\hline 1,200 & & 480 & 11.71 & 650 & 118 & 65 \\
\hline 1,400 & & 491 & 1199 & 662 & 1205 & 66 \\
\hline 1,600 & & 503 & 1226 & 673 & 123 & 675 \\
\hline 1,800 & . & 5.13 & 1253 & 683 & 1255 & 685 \\
\hline 2,000 & & 524 & 1279 & 692 & 128 & 695 \\
\hline 2,200 & & 534 & 1303 & 7.02 & 130 & 70 \\
\hline 2,400 . & . & 544 & 1327 & 7.12 & 132 & 71 \\
\hline 2,600 & . & 5.54 & 1350 & 721 & 1345 & 7.2 \\
\hline 2,800 & . & 5.63 & 13.64 & 730 & 137 & 725 \\
\hline 2,898 & r & 568 & 1364 & 730 & . & \\
\hline 2,900 & &. & & & 13.7 & 72 \\
\hline 2,920 & . & & r. & . & 1365 & 72 \\
\hline
\end{tabular}

upper layer, and $\lambda=\mu$ is assumed. The discontinuity at the interface is replaced by a transition zone as discussed above. The first approximation comes out to be the same as in table 1 of Jeffreys' paper. Since he considered the interface as a discontinuity, our result justifies the replacement of the discontinuity by a transitional zone. As Jeffreys said in his paper, the approximation in his table 1 is unsatisfactory. For example, $C / \beta$ in his table 1 is 1.060 for $k T=3.0$ ( $\beta$ is shear velocity and $T$ is the thickness of the upper layer), whereas the exact value for this case shown in his table 2 is about 1.00, the relative error being about 6 per cent. Although this is the worst error in Jeffreys' example, it is clear that we must refine our first approximation by going to second and third approximations. In fact, the second and third approximations for $k T=3.0$ in the foregoing example give relative errors of 
3 and 1 per cent, respectively. Now our method of approximation gives exact values of $C$ for $k T=0$ and $\infty$. Also, the foregoing example is the least favorable one for our method, since the whole vertical variation in density and elasticities is concentrated at one interface instead of a transition zone, contrary to our assumption just after (4). It appears safe to say that our approximate method will give results sufficiently accurate for our purposes in the cases to be studied in the next section.

\section{Mantle Rayleigh Waves}

Using the method in the last section, we now calculate theoretical dispersion curves of mantle Rayleigh waves for two models of the internal constitution of the earth. In table 1 are shown density distribution by K. E. Bullen and $\mathrm{P}$ and $\mathrm{S}$ wave velocity distributions by B. Gutenberg and H. Jeffreys. Numerical data for the JeffreysBullen distributions can be found in Bullen's book (1953) or in an article by Bullard (1953). Numerical data for Gutenberg's distributions are to be found in a paper by Bullard (1957). A figure is given in Gutenberg's paper (1958) showing his velocity distributions.

As density distribution is known to be of minor importance in determining the dispersion curve, we use Bullen's density distribution in table 1 throughout the following computations. The two velocity distributions in table 1 differ but little for depths greater than $400 \mathrm{~km}$. For these depths we therefore use the arithmetical mean of the corresponding two velocity values. We assume a crust of thickness 35 $\mathrm{km}$., density $2.8 \mathrm{gm} / \mathrm{cm}^{3}$, shear-wave velocity $3.535 \mathrm{~km} / \mathrm{sec}$, , and with $\lambda=\mu$. Immediately under the Mohorovičić discontinuity we assume density $=3.3$ $\mathrm{gm} / \mathrm{cm}^{3}$, shear-wave velocity $=4.7 \mathrm{~km} / \mathrm{sec}$, and compressional-wave velocity $=$ $8.2 \mathrm{~km} / \mathrm{sec}$. In order to get smooth distributions we disregard the velocity values at $60 \mathrm{~km}$. in Gutenberg's distribution. In Jeffreys' distribution we replace the velocities above $200 \mathrm{~km}$. depth with values more in keeping with recent measurements. We take shear-wave velocity $=4.7 \mathrm{~km} / \mathrm{sec}$. and compressional wave velocity = $8.2 \mathrm{~km} / \mathrm{sec}$. between the M-discontinuity and a depth of $200 \mathrm{~km}$. If we need values of $\rho, \lambda$, and $\mu$ at depths not shown in table 1, we make linear interpolations from the corresponding values at the nearest two depths. Thus we have two models for calculating dispersion curves. In the Gutenberg model we have a low-velocity layer at a depth of about $150 \mathrm{~km}$., whereas in the modified Jeffreys model we have no such low-velocity layer. A feature common to these two models is a rather sharp increase in body-wave velocity between 400 and $1,000 \mathrm{~km}$. depth.

The most tedious part of our method is the calculation of the left-hand side of (4). First, we get explicit expressions for the parts depending only on $k z$ in the integrands in (4). For $\vec{u}=\vec{u}(1, k z)=\delta \vec{u}$, for example, we have

$$
\begin{aligned}
\rho \text { part } & =1.71823350 e^{-2 \alpha_{0} k z}-3.64273434 e^{-\left(\alpha_{0}+\beta_{0}\right) k z}+2.48803382 e^{-2 \beta_{0} k z}, \\
-\lambda \text { part } & =0.079392354 e^{-2 \alpha_{0} k z} \\
-\mu \text { part } & =5.90465272 e^{-2 \alpha_{0} k z}-9.71395828 e^{-\left(\alpha_{0}+\beta_{0}\right) k z}+4.20626733 e^{-2 \beta_{0} k z}
\end{aligned}
$$

By the $-\mu$ part, for example, we mean the $\{\quad\}$ in the third integral in (4). Next we calculate these expressions for a range of values of $k z$. These results can be used 
repeatedly for different distributions of density and elasticities as well as for different values of $k$ in the same distribution. In programming the calculations for an electronic digital computor these expressions need be evaluated once and can then stand permanently. For each value of $k$ we next calculate the corresponding distributions of $\rho, \lambda$, and $\mu$ in $k z$ space. Numerical integrations finally give a secular equation to determine $C^{2}$. In our calculation we replace the upper limit $k z=+\infty$ in the integrals by $k z=20$, since for $k z>20$ the integrands are negligibly small. We divide the remaining $\mathrm{kz}$ region into 40 parts, not necessarily of equal interval, and use Simpson's method of numerical integration. Thus we have for $1 / k=200$ $\mathrm{km}$. in the Gutenberg model, for example, $C=5.121,4.987$, and $4.950 \mathrm{~km} / \mathrm{sec}$. in

TABLE 2

\begin{tabular}{|c|c|c|c|c|c|c|}
\hline & \multirow{2}{*}{$1 / k$} & \multirow{2}{*}{ Wave length } & \multicolumn{2}{|c|}{ Gutenberg model } & \multicolumn{2}{|c|}{ Modified Jeffreys model } \\
\hline & & & $C$ & $T$ & $C$ & $T$ \\
\hline \multicolumn{2}{|r|}{$\mathrm{km}$. } & $\mathrm{km}$. & $\mathrm{km} / \mathrm{sec}$ & sec. & $\mathrm{km} / \mathrm{sec}$. & sec. \\
\hline 0 & $\cdots$ & 0 & 3250 & 0 & 3.250 & 0 \\
\hline 17.5 & . & 110 & 3869 & 28.4 & 3920 & 281 \\
\hline 50 . & & 314 & 4107 & 765 & 4331 & 72.5 \\
\hline 70 & & 440 & 4.228 & 104 & 4398 & 100 \\
\hline 100 & . & 628 & 4.306 & 146 & 4.460 & 141 \\
\hline 140. & & 880 & 4.541 & 194 & 4.679 & 188 \\
\hline 175 & $\ldots$ & 1,100 & 4,781 & 230 & 4900 & 225 \\
\hline 200 & .. & 1,256 & 4.950 & 254 & 5052 & 249 \\
\hline 300. & $\ldots \quad \ldots$ & 1,885 & 5610 & 336 & 5610 & 336 \\
\hline & & 2,424 & 606 & 400 & 606 & 400 \\
\hline
\end{tabular}

the first, second, and third approximations, respectively. The further the approximation the smaller $C$ we find. This, of course, follows from a feature of Rayleigh's method in which the approximate values are always larger than the corresponding exact value. The foregoing typical example also shows how rapidly the successive approximations converge. 'The third approximation in the example is considered to be exact to 1 per cent or less positive error. In table 2 are shown all the computed values of $C$ to the third approximation. The value of $C$ for $T=0$ is obtained by multiplying the shear-wave velocity in the crust $=3.535 \mathrm{~km} / \mathrm{sec}$. by the wellknown coefficient 0.9194 . The value of $C$ for the wave length $=2,424 \mathrm{~km}$. is calculated by a method, to be explained later, which involves consideration of spherical curvature of the earth. These results are shown in figure 1 in solid lines. The smooth continuation of the results with and without allowance for curvature provides further assurance of the validity of our method of calculation.

The dispersion of mantle Rayleigh waves has been studied experimentally in great detail since the introduction of modern long-period seismographs (see Benioff and Press, 1958, for a review). In order to compare our theoretical phase velocities with the observed data, we first transform the experimental group-velocity curve into the corresponding phase-velocity curves. Phase velocities are more directly 
related to the internal constitution of the earth than group velocities. ${ }^{1}$ Experimentally determined group velocity $U$ as a function of period $T$ is shown in table 3 . Next, using the formula

$$
\frac{d C}{d T}=\frac{C(C-U)}{U T}
$$

and assuming several initial values, $C=4.00,4.10$, and $4.20 \mathrm{~km} / \mathrm{sec}$. for $T=100$ sec., we integrate (12) numerically and get the results shown in table 3 and figure 1 (dashed curves). It is interesting that the values of $C$ in table 3 for a fixed $T$ change almost linearly with changes in the initial values of $C$ at $T=100$ sec. Thus the values of $C$ corresponding to initial $C=4.10 \mathrm{~km} / \mathrm{sec}$. are almost equal to the mean

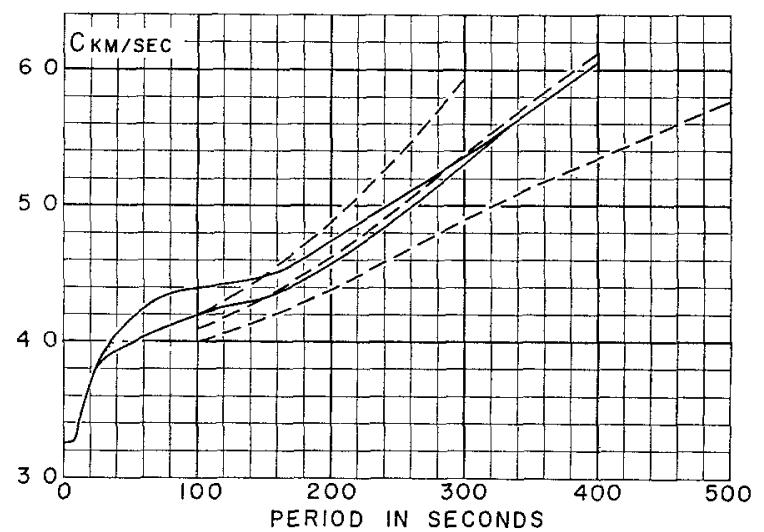

Fig. 1. Comparison of experimental (dashed lines) and theoretical (heavy lines) phase velocity curves for mantle Rayleigh waves.

values of $C$ corresponding to initial $C=4.00$ and $4.20 \mathrm{~km} / \mathrm{sec}$. By linear interpolation we can therefore easily infer $C$ for other assumed initial values of $C$ at $T=100$ sec.

An examination of figure 1 shows that we get good agreement between our theoretical results and an "observed" dispersion curve corresponding to $C=4.10$ $\mathrm{km} /$ sec. at $T=100$ sec. More precisely, if we make a linear interpolation between the results corresponding to $C=4.00$ and 4.10 at $T=100$ sec. and draw a curve corresponding to $C=4.09 \mathrm{~km} /$ sec. at $T=100$ sec., we get good agreement between the trend of the observed phase velocity and the theoretical results based on the Gutenberg model for periods longer than 200 sec. For these periods the modified Jeffreys model gives good agreement, although not quite so good as the Gutenberg model. This suggests that the minimum group velocity which is observed at about 230 sec. is mainly due to the sharp increase of shear-wave velocity at about $400 \mathrm{~km}$. depth which is the feature common to the two models. Wave length corresponding to $T=250$ sec. is about $250 \times 5=1,250 \mathrm{~km}$. Wave length to effective depth ratio here is about 3 , the scale ratio found by one of us in a previous study on the torsional oscillations of the earth (Takeuchi, 1959). For periods shorter than 200 sec., the trend of the modified Jeffreys model departs significantly from the empirical

\footnotetext{
${ }^{1}$ A simple extension of our method permits the direct evaluation of group velocity to the same degree of precision as phase velocity. The numerical work is slightly less than that required in the phase-velocity computation.
} 
curves, whereas that of the Gutenberg model does not. We take this as indicative of a low-velocity layer in the upper mantle. Since mantle Rayleigh-wave dispersion is determined for mixed continental and oceanic paths, the low-velocity zone must be present under continents and oceans. Although some disagreement occurs between the empirical curves and the theoretical curve for the Gutenberg model, this is small and can be reduced even further by assuming that the low-velocity zone begins at a shallower depth. For periods shorter than 25 sec. the Gutenberg and the modified Jeffreys model give almost the same theoretical dispersion curve, since the same density and elasticities in the crust were assumed for both models. Wave length to effective depth ratio is $25 \times 3.8 \mathrm{~km}$. divided by $35 \mathrm{~km}$., or about 3 , as before. By a dotted curve in figure 1 is shown the phase-velocity curve for crustal thickness $35 \mathrm{~km}$. used in studies of crustal structure from phase velocity by one of

TABLE 3

\begin{tabular}{l|cc|cc}
\hline \multirow{2}{*}{$T$} & \multicolumn{3}{|c|}{$U$} & \multicolumn{2}{c}{$c$} \\
\hline & km/sec. & $\mathrm{km} / \mathrm{sec}$. & $\mathrm{km} / \mathrm{sec}$. & $\mathrm{km} / \mathrm{sec}$. \\
100 & 376 & 400 & 410 & 420 \\
150 & 366 & 4159 & 4.324 & 4.493 \\
200 & 359 & 4374 & 4621 & 4.883 \\
250 & 358 & 4631 & 4984 & 5372 \\
300 & 373 & 4902 & 5.385 & 5943 \\
350 & 4.00 & 5.135 & 5.766 & \\
400 & 410 & 5.335 & 6.127 & \\
450 & 412 & 5548 & 6529 & \\
500 & 412 & 5771 & 6.970 & \\
\hline
\end{tabular}

us (Press, 1956). This curve was determined such that it is related to the experimental group-velocity curve for North America and Africa in the period range 10-40 sec. Outside this period range it is related to a theoretical group-velocity curve for a crustal layer of $35 \mathrm{~km}$. thickness with shear velocity $3.51 \mathrm{~km} / \mathrm{sec}$. overlying a homogeneous mantle with shear velocity $4.68 \mathrm{~km} / \mathrm{sec}$. Although this is partly an experimental curve, the theoretical model used to fix the curve is very similar to the two models used in our calculation, especially in crustal structure. In the modified Jeffreys model we assumed shear velocity $=4.7 \mathrm{~km} / \mathrm{sec}$. and compressional wave velocity $=8.2 \mathrm{~km} / \mathrm{sec}$. between $200 \mathrm{~km}$. depth and the M-discontinuity, whereas in the Gutenberg model we assumed a low-velocity layer at about $150 \mathrm{~km}$. Thus the dotted curve in figure 1 falls between the two theoretical curves and nearer to that for the modified Jeffreys case. If, however, our conclusion on the existence of a low-velocity layer is correct, the experimental phase-velocity curve will follow the theoretical curve for the Gutenberg model. Since most experimental phasevelocity determinations have been made for periods less than 30 sec. (Ewing and Press, 1959), the low-velocity zone is not a factor in the use of these data to deduce crustal thickness. It is now important to determine phase velocities directly for periods greater than $40 \mathrm{sec}$. by tripartite array methods. This will provide an independent check on the existence of a low-velocity zone in the upper mantle, and will enable us to surmise more precisely the extent of the zone. 
We now explain how we get $C=6.06 \mathrm{~km} / \mathrm{sec}$. for $T=400 \mathrm{sec}$. (wave length $2,424 \mathrm{~km}$.) in table 2 . It is clear that we are involved with the problem of dilatational oscillations in a sphere with radially variable density and elasticities. N. Jobert (1957) computed a period of $66 \mathrm{~min}$. for the oscillation $S_{2}$, using Bullen's model for the internal constitution, neglecting the self-gravitation of the earth. Pekeris and Jarosch (1958) found the period to be $52 \mathrm{~min}$., allowing for gravity. The fundamental equations in studying this problem can be found in Pekeris and Jarosch's paper. The equations can also be obtained by putting

$$
\begin{array}{ll}
r^{n-1} F_{n}=V, \quad n r^{n-1} F_{n}+r_{0}^{n+1} G_{n}=U, \\
f_{n} r^{n}=X, \quad K_{n} r^{n}=P
\end{array}
$$

in a paper by one of the authors ('Takeuchi, 1950). Pekeris and Jarosch's variational equation 57 is very effective in calculating the periods of free oscillations of the earth. $U, V$, and $P$ in their notation are respectively radial and lateral displacement and an additional potential caused by the deformation of the earth. Fach is proportional to $e^{2 \sigma t}$ and their angular variations are related to a surface spherical harmonic $S_{n}$. In calculating the periods of the earth oscillations of $S_{n}$ type with $n=16$ (wave length $=2,424 \mathrm{~km}$.) we may neglect the self-gravitation of the earth. The calculation proceeds as follows. First put $P=0$ and assume

$$
\begin{aligned}
& U=y^{n-1}\left(n+A y^{2}+B y^{4}\right), \\
& V=y^{n-1}\left(1+D y^{2}+F y^{4}\right), \quad P=a y
\end{aligned}
$$

which is a generalization of eq. 81 in the Pekeris-Jarosch paper. The coefficients of the $y^{n-1}$ terms are chosen so as to annul the $y^{n-2}$ term in $X$. By the boundary conditions (eqs. 53 and 54, Pekeris-Jarosch) we get two relations among $1, A, B, D$, and $F$. Putting these relations into (14) will give expressions for $U$ and $V$ which may be grouped in terms under $1, F$, and $B . \vec{u}=(U, V)$ under the coefficients $1, F$, and $B$ will satisfy the boundary conditions at the free surface $r=a$ separately. Denoting $1, F$, and $B$ by $E_{1}, E_{2}$, and $E_{3}$, respectively, and denoting $\vec{u}$ under $E_{m}$ by $\vec{u}(m, y)$, we have

$$
\vec{u}=\sum_{m} E_{M} \vec{u}(m, y)
$$

From here the procedure is the same as used earlier and leads to $m$ simultaneous linear equations for $E_{m}$. The coefficient of $E_{m}$ in the $m$ 'th equation will be the lefthand side of Pekeris and Jarosch eq. 57 with $\vec{u}$ and $\delta \vec{u}$ replaced by $\vec{u}(m, y)$ and $\vec{u}\left(m^{\prime}, y\right)$, respectively. Putting the determinant formed by the coefficients equal to zero results in an equation for $\sigma^{2}$.

The first, second, and third approximations for $n=16$ give $T=6.0,6.5$, and $6.67 \mathrm{~min}$., respectively. The convergency here is fairly good. It is this third approximation which gives $C=6.06 \mathrm{~km} /$ sec. for $T=400 \mathrm{sec}$. (wave length $=2,424 \mathrm{~km}$.) in table 2 . 


\section{ACKNOWLEDGMENTS}

The authors thank Dr. Gutenberg for his helpful suggestions in the course of this study, Dr. N. Jobert for her discussions by mail, and the Alfred P. Sloan Foundation for financial support.

\section{REFERENCES}

Benioff, H., and F. Press

1958. "Progress Report on Long-Period Seismographs," Geophys. Jour., 1: 208-215.

Bullard, E. C.

1953. The Earth as a Planet (Univ. of Chicago Press), pp. 57-137.

1957. "The Density within the Earth," Verhandelingen van het Koninklijk Nederlandsch Geologisch-Minjnbouwwkundig Genootschap, Geologische Serie, Deel 18, Gedenkboek F. A. Vening Meinesz.

Bullen, $\mathrm{K} \mathrm{E}$.

1953. An Introduction to the Theory of Seismology, 2d ed. (Cambridge Univ. Press), pp. 218-220.

Ewing, M., and F. Press

1959. "Determination of Crustal Structure from Phase Velocity of Rayleigh Waves, Part 3: The United States," Bull. Geol. Soc. Am., 70: 229-244.

Ewing, W. M., W.S Jardetzky, and F. Press

1957. Elastic Waves in Layered Media (New York: McGraw-Hill), 380 pp.

Gutenberg, B.

1953. "Wave Velocities at Depths between 50 and 600 Kilometers," Bull. Sersm. Soc. Am., 43: 223-232.

1958. "Velocity of Seismic Waves in the Earth's Mantle," Trans. Am. Geophys. Union, 39: $486-489$.

Jeffreys, $\mathrm{H}$.

1934. "The Surface Waves of Earthquakes," Mon. Not. Roy. Astron. Soc., Geophys. Suppl., 3: $253-261$.

Jobert, N.

1957. "Sur la période propre des oscillations sphéroidales de la terre," Comptes Rendus, 244: 921-922.

Landisman, M., and Y. Satô

1958. "Shear-Wave Velocities in the Upper Mantle," Abstr. Trans. Am. Geophys. Union, 39: $522-523$.

Pekeris, C. L., and H. Jarosch

1958. "The Free Oscillations of the Earth," Contributions in Geophysics in Honor of B. Gutenberg (London: Pergamon Press), pp. 171-192.

Press, F.

1956. "Determination of Crustal Structure from Phase Velocity of Rayleigh Waves, Part 1: Southern California," Bull. Geol Soc. Am., 67: 1647-1658.

1959. "Some Implications on Mantle and Crustal Structure from G-Waves and Love Waves," Jour. Geophys. Res., 64: 565-568.

Press, F., and M. Ewing

1956. "A Mechanism for G-Wave Propagation," Abstr. Trans. Am. Geophys. Union, 37: 355356.

Takeuchi, H.

1950. "On the Earth Tide of the Compressible Earth of Variable Density and Elasticity," Trans. Am. Geophys. Union, 31: 651-689.

1959. "Torsional Oscillations of the Earth and Some Related Problems," Geophys. Jour , 2: 89-100.

California Institute of Technology,

Pasadena, California.

(Division of the Geological Sciences, contribution no. 922.) 